\title{
Study of serum gamma glutamyl transferase as a diagnostic marker in alcoholic hepatitis
}

\author{
${ }^{1}$ Dr.J.b gogoi, ${ }^{2}$ Dr. Praveen k.Tyagi, ${ }^{3}$ Dr. Amit k.Singh, ${ }^{4}$ Dr. Kailash gairola \\ ${ }^{1}$ Dr Javin Bishnu Gogoi, MBBS, MD (Biochemistry)Associate professor cum HOD, Dept of Biochemistry, VCSG \\ Govt Medical Science \& Research Institute,Srikot, Srinagar (Pauri), Uttarakhand, India, Pin-246174 \\ ${ }^{2}$ Dr Praveen K. Tyagi, MBBS, MS (General Surgery)Assistant professor, Dept of General Surgery, VCSG Govt \\ Medical Science \& Research Institute,Srikot, Srinagar (Pauri), Uttarakhand, India, Pin-246174 \\ ${ }^{3}$ Dr. Amit Kumar Singh, MBBS, MD (Community Medicine)Associate professor, Dept of Community \\ Medicine,VCSG Govt Medical Science \& Research Institute,Srikot, Srinagar (Pauri), \\ Uttarakhand, India, Pin-246174 \\ ${ }^{4}$ Dr Kailash Gairola, MBBS,MD (Biochemistry)Senior Demonstrator, Dept of Biochemistry, VCSG Govt \\ Medical Science \& Research Institute,Srikot, Srinagar (Pauri), Uttarakhand, India, Pin-246174
}

\begin{abstract}
Serum gamma glutamyl transferase (GGT or $\gamma$-GT) is a microsomal enzyme, catalyzes the transfer of the gamma-glutamyl moiety of glutathione to various peptide acceptors. It is a reliable diagnostic marker of alcohol ingestion which is one of the most sensitive and most commonly employed biochemical marker of alcohol consumption . This study was carried out with 30 alcoholic hepatitis and 30 normal non-alcoholic control subjects. The aim of this study was to establish the role of serum GGT alone in the diagnosis of alcoholic hepatitis. A significant correlation in the level of serum GGT is observed in alcoholics when compared with the normal subjects. The enzyme activity of GGT in serum is increased in average by $\mathbf{5 2 7 . 3 1 \%}$ in the patients with alcoholic hepatitis compared to normal control subjects $(\mathbf{p}<0.05)$. Measurement of GGT in serum appears to be a sensitive index with low specificity in the diagnosis of alcoholics with hepatitis when correlated clinically.
\end{abstract}

Keywords-Alcoholic hepatitis, Gamma glutamyl transferase or transpeptidase (GGT or $\gamma$-GT), Alcoholics.

\section{INTRODUCTION}

Gamma glutamyl transferase or transpeptidase (GGT or $\gamma$-GT) is a microsomal enzyme with a wide tissue distribution. It is a membrane-bound glycoprotein enzyme, catalyzes the transfer of the gamma-glutamyl moiety of glutathione to various peptide acceptors. However serum GGT is an enzyme having special relation with alcoholic liver diseases. GGT is a widely used clinical marker of alcohol abuse ${ }^{1}$. This biliary canalicular enzyme is induced by alcohols and its serum level rises in response to acute hepatocellular damage ${ }^{2}$.

Alcoholism ranks as one of the main current threats to the health and safety of people in most countries of the world. Chronic alcoholism is considered as a social disease or health related problem inflicting a good sum of population globally. Alcoholic liver disease is a leading cause of morbidity and mortality worldwide ${ }^{3}$. Therefore, a high priority should be given to aim at reducing its prevalence through more effective diagnosis and early intervention. The need for objective methods for revealing alcohol abuse in its early phase has also been widely acknowledged. ${ }^{4}$

A variety of liver enzymes assist in the diagnosis of alcohol addiction; including, gamma-glutamyl transferase (GGT), the most sensitive and widely employed marker, alanine aminotransferase (ALT) and aspartate aminotransferase (AST). GGT is one of the longest established biochemical tests for excessive alcohol consumption. GGT is an enzyme in bile, which is induced to rise by alcohol and serum levels due to liver damage $e^{2,5}$.

\section{METHODS AND MATERIALS}

The present study was carried out with 60 cases out of which 30 cases were established alcoholic hepatitis patients. The control group (30 cases) was selected from non alcoholic normal, healthy male volunteers. Blood samples were collected from the two groups for determination of the activity of GGT in serum. The patients selected for the study were free from infective hepatitis as well as the samples were collected before the start of treatment to prevent any change of GGT due to drugs administration.

Inclusion criteria:

(1) History of alcoholism 
(2) Clinical and biochemical evidences of hepatic abnormality

(3) Ultrasonographic evidence

(4) Histopathological examination

\section{Exclusion criteria-}

1. Infective hepatitis cases

2. Alcoholic cases who has started treatment

The results were analyzed statistically by student's $t$ test.

\section{RESULTS AND DISCUSSION}

In this chapter an effort would be made to correlate the present findings with the results of the previous workers and to discuss the subject as a whole.

The serum GGT level in alcoholic hepatitis group was compared to the activity of this enzyme in 30 normal controls.

The results are represented in table-1

\begin{tabular}{|l|l|l|}
\hline GROUP & $\begin{array}{l}\text { SERUM GGT U/L } \\
\text { (average value) }\end{array}$ & $\begin{array}{l}\text { SD } \\
\pm\end{array}$ \\
\hline ALCOHOLIC HEPATITIS $(\mathrm{n}=30)$ & 107.73 & 10.45 \\
\hline NORMAL $(\mathrm{n}=30)$ & 20.43 & 7.72 \\
\hline
\end{tabular}

Bar diagram 1:-Showing Serum GGT (average value in U/L) in Alcoholic hepatitis and control group

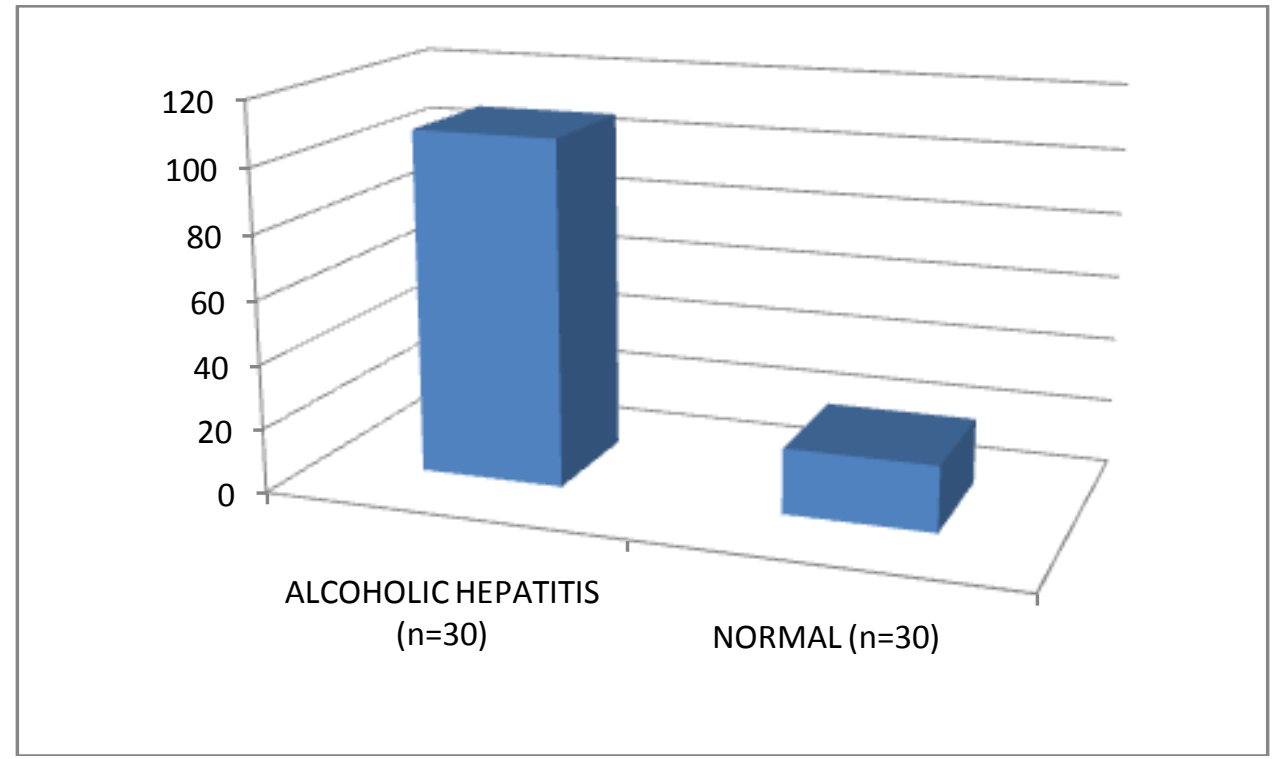

The average normal serum GGT level is $20.43 \pm 7.72 \mathrm{U} / \mathrm{L}$ in the control group. As against this value in alcoholic hepatitis, the increase in serum GGT is seen by $527.31 \%(\mathrm{p}<0.05)$ with an average value of 107.73 $\pm 10.45 \mathrm{U} / \mathrm{L}$

GGT is extremely sensitive to alcohol use and serum GGT is one of the best markers for chronic alcohol consumption, which has a relatively high sensitivity and specificity ${ }^{6}$. When the increase in GGT is two or more times greater than the increase in ALP, the source of the ALP is considered to be from the liver. When the increase in GGT is five or more times greater than the increase in ALP, this point to a diagnosis of alcoholic hepatitis. ${ }^{7}$ The serum level of this enzyme gets elevated in cholestasis and hepatobiliary disorder and by drugs including alcohol possibly due to enzyme induction. Levels parallels serum ALP in cholestasis and may be used to confirm that a raised serum ALP is of hepato biliary origin ${ }^{8}$. Especially high levels of serum GGT are seen in patients with severe alcoholic liver disease though they may fall in the later stages of cirrhosis. ${ }^{9}$ The serum GGT is markedly elevated in patients with alcoholic liver disease and primary biliary cirrhosis whereas mean hepatic GGT is significantly elevated only in the alcoholic liver disease group. ${ }^{\mathbf{1 0}}$ Serum GGT is the most sensitive, most widely employed marker of alcohol consumption. It is more likely to be elevated in regular rather than episodic drinkers. ${ }^{3,11}$ An isolated rise in serum GGT is seen in patients with alcohol abuse, even without liver disease, 
perhaps because of microsomal enzyme induction. More often there is steatosis. In fibrosis, cirrhosis and hepatitis due to alcohol, other liver enzymes are elevated in conjunction with GGT. ${ }^{12}$ Matsuda et al in 1993 classified GGT responses to alcoholic drinking into 3 groups: non response, mild response and hyper response. In alcoholic liver disease non responders were scarcely found and the response of GGT tended to increase in parallel with progression of liver disease. ${ }^{13}$ Still some workers find that assay of GGT in serum is a misleading test and measurement of hepatic mitochondrial AST as a new marker enzyme in chronic alcoholism. However, the results of the present investigation signify the importance of measurement of serum GGT as a diagnostic marker in alcoholic hepatitis.

There are no reliable markers to detect heavy drinking or as a tool to control abstinence compliance in alcoholic treatments. The Mean Corpuscular Volume (MCV), and the gammaglutamyl transpeptidase (GGT), are widely used although their predictive value is somewhat limited due to their low specificity. ${ }^{14}$

This marker may be very useful in opportunistic case finding, in motivating patients to change drinking habit and in monitoring the treatment response. ${ }^{5}$

\section{CONCLUSION}

This conventional test is widely available and relatively inexpensive. Though having limited sensitivity and specificity in detection of excessive drinking, it also provide valuable data on complications of drinking and comorbid conditions that may be affected by drinking and, in some cases, prognosis. This study thus is indicative of hepatic microsomal injury in chronic alcoholics supporting the findings of earlier workers. Hence, determination of serum GGT in alcoholic hepatitis will be a useful diagnostic tool if used judiciously and correlated clinically. Also to be noted that estimation of GGT is more informative in detecting alcoholism if it is used along with other biochemical parameters like ALT, AST, ALP, MCV, etc.

\section{REFERENCES}

[1]. Puukka K, Hietala J, Koivisto H, Anttila P, Bloigu R, Niemelä O. Obesity and the clinical use of serum GGT activity as a marker of heavy drinking. Scand J Clin Lab Invest. 2007; 67 (5):480-8.)

[2]. Subir Kumar Das, Prasunpriya Nayak and D.M.Vasudevan. Biochemical markers for alcohol consumption. IJCB, 2003, 18(2) 111-118.

[3]. J.B. Gogoi, S.K. Gupta, P.K. Mishra, B. Karunanand. Alcohol abuse and alcoholic hepatitis in upper Assam. Indian J. Prev. Soc. Med. Vol 39 No. $1 \& 2,2008 ; 28-29$.

[4]. Niemelä O. Biomarkers in alcoholism. Clin Chim Acta. 2007 Feb; 377(1-2):39-49.)

[5]. Conigrave, KM, Davies, P., Haber, P. and Whitfield, JB. Traditional markers of excessive alcohol use. Addiction, 2003; 98: 3143

[6]. Bosma PJ, Chowdhury JR, Bakker C, Gantla S, de Boer A, Oostra BA, et al. The genetic basis of the reduced expression of bilirubin UDP-glucuronosyltransferase 1 in Gilbert's syndrome. N Engl J Med 1995;333:1171-5

[7]. Jennifer Heisler, RN, About.com Updated: March 1, 2009

[8]. Rosalki SB. Gamma - glutamyl Transpeptidase. Adv. Clinical Chemistry. 1975; 17: 53.

[9]. Wu. A., Slavin, G. and Levi, A. J.: Eleveted serum GGT and histological liver damage in alcoholism. Am. J. Gastroenterol 65, 1976:318-323.

[10]. Selinger M. J. et al: "GGT activity in liver disease": ClinChim. Acta: Nov.10, 1982: 125 (3): 283-90.

[11]. Rosalki, S. (1984) - Identifying the alcoholic. In clinical Biochem. of Alcoholism, (Ed. Rosalki S) PP. 65-92. Churchill, Livingstone, Edinburgh.

[12]. Ireland A, Hartley L, Ryley $\mathrm{N}$ et al.: Raised Gamma-Glutamyl-transferase activity and the need for liver biopsy. Br. Med.J.$1991 ; 302: 388$

[13]. Matsuda Y et al: "The relationship between the development of alcoholic liver and pancreatic disease and induction of GGT: Alcohol suppl: 1B: 27-33, 1993.

[14]. Glasinovic JC, Lobos X, Scrivanti M, Severín MC, Quiroga T, Moncada C. Carbohydrate deficient transferrin, gammaglutamyl transferase and mean corpuscular volume in the evaluation of recent alcohol intake in excessive drinkers. Rev Med Chil. 2001 Apr; 129(4):375-81). 